\title{
21. Landkreise und kreisfreie Städte: Regionale Unterschiede im freiwilligen Engagement
}

\author{
Nicole Hameister \& Clemens Tesch-Römer
}

\section{Kernaussagen}

Der Anteil freiwillig engagierter Menschen ist in Deutschland regional ungleich verteilt. Menschen, die im städtischen Raum leben, engagieren sich zu geringeren Anteilen als Menschen, die im ländlichen Raum leben.

Am niedrigsten ist der Anteil Engagierter in Großstädten. In kreisfreien Großstädten beträgt die Engagementquote 39,0 Prozent. Deutlich höher ist die Engagementquote in städtischen Kreisen (45,4 Prozent), in ländlichen Kreisen mit Verdichtungsansätzen (46,0 Prozent) und in dünn besiedelten Landkreisen (45,0 Prozent).

Je besser die Arbeitsmarktlage in einer Region ist, desto höher ist die Engagementquote. In Regionen mit einer niedrigen Arbeitslosenquote ist der Anteil freiwillig Engagierter hoch. In Regionen mit einer hohen Arbeitslosenquote ist der Anteil von freiwillig Engagierten deutlich niedriger.

\subsection{Einleitung}

Engagement entfaltet sich in räumlichen Kontexten. Engagierte Menschen leben auf dem Land oder in der Stadt, sie beteiligen sich in der unmittelbaren Nachbarschaft oder an einem weiter entfernt liegenden Ort, sie treffen sich in Mehrgenerationen-Häusern oder Nachbarschaftsheimen. Der räumliche Kontext ist wichtig, weil er Gelegenheitsstrukturen für freiwilliges Engagement bietet. Orte, an denen Menschen leben, können mit lokalen Angeboten von Initiativen, Vereinen und Verbänden zum Mitmachen einladen. In vielen Kommunen gibt es eine lokale Engagementinfrastruktur, die wohnortnahe und niedrigschwellige Zugänge zum freiwilligen Engagement bietet (Zimmer \& Backhaus-Maul 2012). Gibt es vor Ort eine Vielzahl von Engagementmöglichkeiten, so kann man davon ausgehen, dass sich Menschen mit höherer Wahrscheinlichkeit freiwillig engagieren als in Orten, in denen es an Gelegenheiten zu gemeinschaftlichen Aktivitäten und Mitmachmöglichkeiten mangelt.

Der Einfluss der räumlichen und örtlichen Umwelt kann auf unterschiedlichen Ebenen untersucht werden. Die Bedeutung der Nachbarschaft für freiwilliges Engagement wird im Kapitel 19 betrachtet. Unterschiede zwischen den Bundesländern sowie zwischen Ost- und Westdeutschland werden in Kapitel 22 analysiert. Im vorliegenden Kapitel wird der Frage nachgegangen, welche Bedeutung der kommunale Kontext auf der Ebene von Landkreisen und kreisfreien Städten für das freiwillige Engagement von Menschen hat.

Aus zwei Gründen wurde die Ebene der (Land-)Kreise und kreisfreien Städte für die Analysen in diesem Kapitel gewählt (diese beiden Gründe werden in ähnlicher Weise auch im 
Raumordnungsbericht thematisiert, siehe Bundesinstitut für Bau-, Stadt- und Raumforschung (BBSR) 2012: 17). Erstens hat der kommunale Lebenskontext große Bedeutung für das freiwillige Engagement von Menschen, die dort leben. So ist beispielsweise auf der Ebene der (Land-)Kreise und kreisfreien Städte ein bedeutsamer Teil jener Infrastruktur zu finden, die für das freiwillige Engagement von Bedeutung ist. Aber auch andere räumliche Merkmale von Landkreisen und kreisfreien Städten, wie etwa das Angebot an öffentlichem Personennahverkehr, können sich förderlich oder hinderlich auf das freiwillige Engagement auswirken. Zweitens ist auf der Ebene der (Land-)Kreise und kreisfreien Städte die umfangreichste Datenbasis für die räumliche Beschreibung zu finden. Informationen zu Demografie, Wirtschaft, Arbeitsmarkt, Wohlstand, Infrastruktur und Wohnungsmarkt sind, jährlich aktualisiert, für alle Landkreise und kreisfreien Städte in Deutschland erhältlich (BBSR 2012).

Im Jahr 2014 gab es in Deutschland 295 Landkreise und 107 kreisfreie Städte (insgesamt 402 räumliche Einheiten). Die Zahl der Landkreise und kreisfreien Städte hat sich in den letzten Jahrzehnten verringert, vor allem aufgrund der Gebietsreformen in den neuen Ländern. Ihre Zahl sank von 543 im Jahr 1993 auf 402 im Jahr 2014. Trotz dieses Rückgangs ist die Zahl der Landkreise und kreisfreien Städte zu groß, um individuelle Analysen für alle 402 Kommunen durchzuführen. Daher werden wir Landkreise und kreisfreie Städte in Deutschland zu Gruppen zusammenfassen, und zwar (a) nach den Strukturmerkmalen Stadt oder Land, (b) nach dem siedlungsstrukturellen Kreistyp sowie (c) nach der wirtschaftlichen Prosperität der jeweiligen Region (gemessen anhand der Arbeitslosenquote in der jeweiligen Region). Analysen werden auf der Ebene von Gruppen strukturell ähnlicher Kreise durchgeführt.

Stadt und Land: Der Regionstyp ,Stadt und Land' gibt an, ob es sich um eine städtische oder ländliche Region handelt. Obwohl in der soziologischen Debatte eine klare Stadt-Land-Trennung zugunsten eines Stadt-Land-Kontinuums auf- gegeben worden ist (Strubelt 1998), kann man doch festhalten, dass ein ländlicher Regionstyp eher durch geringe Bevölkerungsdichte und einen höheren Anteil an Landwirtschaft charakterisiert ist als ein städtischer Regionstyp. Ein städtischer Regionstyp verfügt dagegen in der Regel über eine hohe Bevölkerungsdichte sowie über wirtschaftliche Unternehmen, die stärker im Industrie- und Dienstleistungssektor angesiedelt sind als dies in ländlichen Gebieten der Fall ist. In verschiedenen Studien wurde in ländlichen Regionen eine höhere Engagementquote festgestellt als in städtischen Regionen (Gensicke 2015; vgl. auch Brauer 2015; Neu 2007; Neu \& Nikolic 2014; Scherger, Brauer \& Künemund 2004). In der internationalen Literatur wird die Frage, ob es einen Stadt-Land-Unterschied mit Blick auf freiwilliges Engagement gibt, kontroverser diskutiert (Wilson 2000; Wilson 2012). In städtischen Regionen könnte die Zahl und Vielfalt an zivilgesellschaftlichen Organisationen größer sein als auf dem Land. Gleichzeitig kann aber auf dem Land die soziale Verpflichtung, sich freiwillig zu engagieren, höher sein als in der Stadt.

Siedlungsstruktureller Kreistyp: Während die Gegenüberstellung ,Stadt und Land' nur zwei Ausprägungen hat, werden bei den siedlungsstrukturellen Kreistypen vier Kategorien unterschieden: Kreisfreie Großstädte, städtische Kreise, ländliche Kreise mit Verdichtungsansätzen, dünn besiedelte ländliche Kreise. Es handelt sich hierbei um eine Ausdifferenzierung des Gegensatzes ,Stadt und Land' (der städtische Regionstyp umfasst Großstädte und städtische Kreise; der ländliche Regionstyp umfasst ländliche Kreise mit Verdichtungsansätzen sowie dünn besiedelte ländliche Kreise). Anhand des siedlungsstrukturellen Kreistyps ist es möglich, Abstufungen der Dimension städtisch-ländlich $\mathrm{zu}$ unterscheiden. Folgt man den Ergebnissen früherer Wellen des Freiwilligensurveys, könnte man annehmen, dass der Anteil engagierter Menschen höher ist, je ländlicher die räumliche Einheit ist (Gensicke 2015: 25).

Wirtschaftliche Prosperität: Als Indikator für die wirtschaftliche Prosperität von Landkreisen 
und kreisfreien Städten wurde für die vorliegenden Analysen die Arbeitslosenquote gewählt. Dieser Indikator stammt aus der Arbeitsmarktstatistik der Bundesagentur für Arbeit. Die Arbeitslosenquote gibt den Anteil der arbeitslos gemeldeten Personen an den Erwerbspersonen in Prozent an. Ein höherer Anteil Arbeitsloser geht in der Regel mit geringeren Steuereinnahmen und höheren kommunalen Ausgaben einher. Eine niedrige Arbeitslosenquote ist ein Indikator für einen gut ausgelasteten Arbeitsmarkt und höhere Steuereinnahmen. Möglicherweise können Kommunen, die aufgrund eines hohen Beschäftigungsgrades über solide kommunale Finanzen verfügen, eine größere Anzahl von zivilgesellschaftlichen Initiativen, Vereinen und Verbänden unterstützen als es Kreisen mit geringerer finanzieller Ausstattung möglich ist. Empirische Analysen zeigen, dass die ehrenamtliche Betätigung von Menschen in der zweiten Lebenshälfte mit dem regionalen Wohlstand verknüpft ist: Je höher der Wohlstand einer Region, desto höher ist die Beteiligung an ehrenamtlicher Betätigung (Simonson, Hagen, Vogel \& Motel-Klingebiel 2013). Allerdings könnte auch die gegensätzliche Hypothese formuliert werden: $\mathrm{Da}$ in Regionen mit höherer Arbeitslosigkeit ein größerer Bedarf für freiwilliges Engagement besteht, könnte der Anteil von freiwillig Engagierten höher sein als in Regionen mit geringer Arbeitslosigkeit, in denen der Bedarf für freiwilliges Engagement geringer ist.

Die empirischen Analysen dieses Kapitels werden Aufschluss über diese Annahmen erbringen. Allerdings muss dabei auf eine Frage hingewiesen werden, die sich bei der Untersuchung räumlicher Einheiten in grundsätzlicher Weise stellt (Kawachi \& Berkman 2003). Diese Frage lautet: Wenn Unterschiede zwischen räumlichen Einheiten gefunden werden (im vorliegenden Fall Unterschiede im Anteil freiwillig engagierter Menschen), sind diese auf Kontexteffekte oder auf Kompositionseffekte zurückzuführen? Die bisherige Argumentation beruht auf der Überlegung, dass es sich um die Effekte des Kontexts handelt: Merkmale von Landkreisen und kreisfreien Städten sind dafür verantwortlich, dass sich Menschen häufiger oder seltener engagieren. Allerdings ist bekannt, dass sich auch die Zusammensetzung der Bevölkerung in unterschiedlichen Regionstypen unterscheidet. In diesem Fall würde es sich um einen Kompositionseffekt handeln: Da die Bevölkerung auf dem Land möglicherweise anders zusammengesetzt ist als in der Stadt, ist es die Komposition der Bevölkerung, die für die Unterschiede verantwortlich ist (und nicht die Merkmale des Kontextes). Daher ist es notwendig, etwaige Unterschiede zwischen Regionstypen dadurch abzusichern, dass in multivariaten Analysen die Komposition der Bevölkerung (nach Alter, Geschlecht oder Bildungsstatus) berücksichtigt wird.

Im vorliegenden Kapitel gehen wir vier Fragen nach:

1. Unterscheidet sich die Engagementbeteiligung in der Stadt und auf dem Land? Finden sich in städtischen und ländlichen Regionen ähnliche (oder unterschiedliche) Verteilungen des Engagements nach Geschlecht, Altersund Bildungsgruppen?

2. Unterscheidet sich die Engagementbeteiligung nach siedlungsstrukturellen Merkmalen? Finden sich in unterschiedlichen siedlungsstrukturellen Regionstypen ähnliche (oder unterschiedliche) Verteilungen des Engagements nach Geschlecht, Alters- und Bildungsgruppen?

3. Unterscheidet sich die Engagementbeteiligung nach dem wirtschaftlichen Status einer Region? Finden sich in unterschiedlich durch Arbeitslosigkeit betroffenen Landkreisen und kreisfreien Städten ähnliche (oder unterschiedliche) Verteilungen des Engagements nach Geschlecht, Alters- und Bildungsgruppen?

4. Finden sich Unterschiede zwischen Regionstypen auch dann, wenn die individuellen Merkmale der Bewohnerinnen und Bewohner dieser Regionen berücksichtigt werden? 


\subsection{Datengrundlage}

\subsubsection{Erhebungsdaten aus dem Freiwilligensurvey}

Die in diesem Kapitel berichteten deskriptiven Befunde basieren auf gewichteten Analysen. Für die Gewichtung wurden in allen Erhebungsjahren dieselben Gewichtungsmerkmale berücksichtigt. Dabei handelt es sich um die Designgewichtung, die sich auf die Auswahlwahrscheinlichkeit einer Person bezieht (Haushaltsgröße, Zahl der Festnetznummern und, nur für das Jahr 2014, auch die Zahl der Mobilfunknummern) und Gewichtungsmerkmale, die Abweichungen der Stichprobenverteilung von der Grundgesamtheit in bestimmten Dimensionen ausgleichen (Bundesland, Gemeindegrößenklassen, Geschlecht, Altersgruppen). Von einer Hinzunahme weiterer Gewichtungsmerkmale wie etwa des Bildungsstandes haben wir abgesehen, um die Vergleichbarkeit mit den bisher veröffentlichten Berichten zu erhalten.

Freiwilliges Engagement: In diesem Kapitel stellen wir die Angaben zum freiwilligen Engagement mit Blick auf räumliche Einheiten dar. Freiwilliges Engagement umfasst freiwillig übernommene Arbeiten und Aufgaben, die außerhalb von Beruf und Familie unentgeltlich erbracht werden oder gegen eine geringe Aufwandsentschädigung (siehe Kapitel 3).

Einverständnis zum Zuspielen von Regionaldaten: Alle Befragten im Freiwilligensurvey 2014 wurden um ihr Einverständnis zum Zuspielen von Regionaldaten gebeten. Der größte Teil der Befragten gab ihr Einverständnis ( $\mathrm{n}=25.847)$, ein kleiner Teil der Befragten gab dieses Einverständnis nicht $(\mathrm{n}=2.843)$. Die Analysen mit Einbezug des regionalen Kontexts sind nur für diejenigen Befragten möglich, die ihr Einverständnis zum Zusammenfügen ihrer Daten mit
Strukturdaten gegeben haben. ${ }^{1}$ Dadurch werden 2.843 Fälle von den Analysen mit regionalem Kontext ausgeschlossen.

Postleitzahl und Kreiskennziffer: Alle Befragten im Freiwilligensurvey 2014, die ihr Einverständnis zum Zuspielen von externen Regionaldaten gegeben haben, wurden anschließend um die Angabe ihrer Postleitzahl gebeten:

Würden Sie mir bitte zu diesem Zweck sagen, wie die Postleitzahl Ihres Wohnorts lautet?

Aus der Postleitzahl kann die Kreiskennziffer eindeutig abgeleitet werden, die als Schlüssel für das Anspielen der Regionalinformationen notwendig ist. Es können also nur solche Fälle in die Analysen einfließen, denen das Befragungsinstitut aus der Postleitzahl eine gültige Kreiskennziffer zuweisen konnte. Für 372 Personen, die ihre Einwilligung zum Zuspielen von regionalen Daten gegeben hatten, war dies nicht möglich. Es verbleiben also 25.475 Fälle im Datensatz für die Regionalanalysen. Bei der Erhebung des Freiwilligensurveys 2014 konnten in allen 402 Kreisen Personen befragt werden. Die durchschnittliche

1 Die Formulierung zum Einholen des Einverständnisses am Ende des Interviews lautete wie folgt: „Die Lebenssituation der Menschen in Deutschland ist recht unterschiedlich. Deshalb sollen die Ergebnisse der Studie auch regional zugeordnet werden - natürlich in anonymisierter Form. Das Deutsche Zentrum für Altersfragen würde gerne einige regionale Daten mit den jetzt aufgenommenen Interviewdaten $\mathrm{zu}$ sammenführen. Dabei handelt es sich zum Beispiel um das Ausbildungsangebot oder die medizinische Versorgung in einer Region. Dazu benötigen wir Ihre Postleitzahl. Es ist absolut sichergestellt, dass sowohl bei infas als auch beim Deutschen Zentrum für Altersfragen immer alle datenschutzrechtlichen Bestimmungen strengstens eingehalten werden. Zum Zweck der Zuspielung dieser Daten an die Interviewdaten möchte ich Sie herzlich um Ihr Einverständnis bitten. Ihr Einverständnis ist selbstverständlich freiwillig. Sie können es auch jederzeit wieder zurückziehen. Sind Sie damit einverstanden?" 
Anzahl der Befragten pro Kreis liegt bei 63,4 (Minimum fünf befragte Personen, Maximum 1.732 befragte Personen ${ }^{2}$ ).

Zusätzliche Gewichtung für die Regionalanalysen: Diejenigen Personen, die ihre Zustimmung zum Zuspielen der Regionaldaten nicht explizit gegeben haben, wurden von den Analysen mit regionalen Zusatzinformationen ausgeschlossen. Diese Ausfälle sind nicht komplett zufällig über alle Befragten verteilt: Die jüngsten und die ältesten Befragten, Frauen, Niedriggebildete und Teilnehmerinnen und Teilnehmer in der Mobilfunkstichprobe geben etwas seltener ihre Zustimmung zur Zuspielung von Regionaldaten. Für die Gemeindegrößenklasse oder die Bundeslandzugehörigkeit lassen sich nur sehr vereinzelt Zusammenhänge mit der Zustimmung zur Zuspielung regionaler Daten finden. Um Verzerrungen aufgrund der unterschiedlichen Zustimmungsraten auszugleichen und damit die Vergleichbarkeit der Regionalanalysen mit den Analysen der gesamten Stichprobe zu erreichen, wird für alle deskriptiven Analysen in diesem Kapitel ein zusätzliches Ausfallgewicht verwendet. Dieses zusätzliche Ausfallgewicht passt die Verteilung der Regionalstichprobe an die Verteilung der Gesamtstichprobe des Freiwilligensurveys an.

2 Der Maximalwert von 1.732 Befragten stammt aus Berlin (Hamburg: 1.565, Bremen: 991). Die Stadtstaaten entsprechen jeweils einem Kreis.

\subsubsection{Regionaldaten des Bundes- instituts für Bau-, Stadt- und Raumforschung (BBSR)}

Um den Zusammenhang zwischen regionalem Kontext und Engagement analysieren zu können, wurden den Befragungsdaten des Freiwilligensurveys 2014 regionale Zusatzinformationen zugespielt. Für jede Person, die ihre Einwilligung für die Zuspielung regionaler Daten gegeben hatte, wurde angegeben, in welchem Regionstyp (städtisch oder ländlich) und in welchem Siedlungsstrukturtyp sie lebt. Zudem wurde vermerkt, wie hoch die Arbeitslosenquote in dem Landkreis beziehungsweise in der kreisfreien Stadt ist, in dem beziehungsweise in der die jeweilige Person lebt. Auf diese Weise ist es möglich, den Zusammenhang zwischen räumlichen Kontexten und individuellen Merkmalen $\mathrm{zu}$ analysieren.

Das Bundesinstitut für Bau-, Stadt- und Raumforschung (BBSR) stellt für Forschungszwecke aggregierte Indikatoren zu verschiedenen Themenbereichen zur Verfügung. Diese strukturellen Merkmale können über einen bestimmten Regionalschlüssel, im vorliegenden Fall die Kreiskennziffer, an Datensätze aus anderen Quellen angespielt werden. Die in diesem Kapitel verwendeten Indikatoren des BBSR basieren auf dem Gebietsstand vom 31. Dezember 2012. Es ist davon auszugehen, dass diese regionalen Indikatoren im Zeitraum von 2012 bis 2014 sehr stabil geblieben sind. Zunächst stellen wir in diesem Abschnitt dar, wie sich die verwendeten Indikatoren über die Kreise in Deutschland verteilen. 


\section{Abbildung 21-1: Verteilung von städtischen und ländlichen Regionstypen in Deutschland}

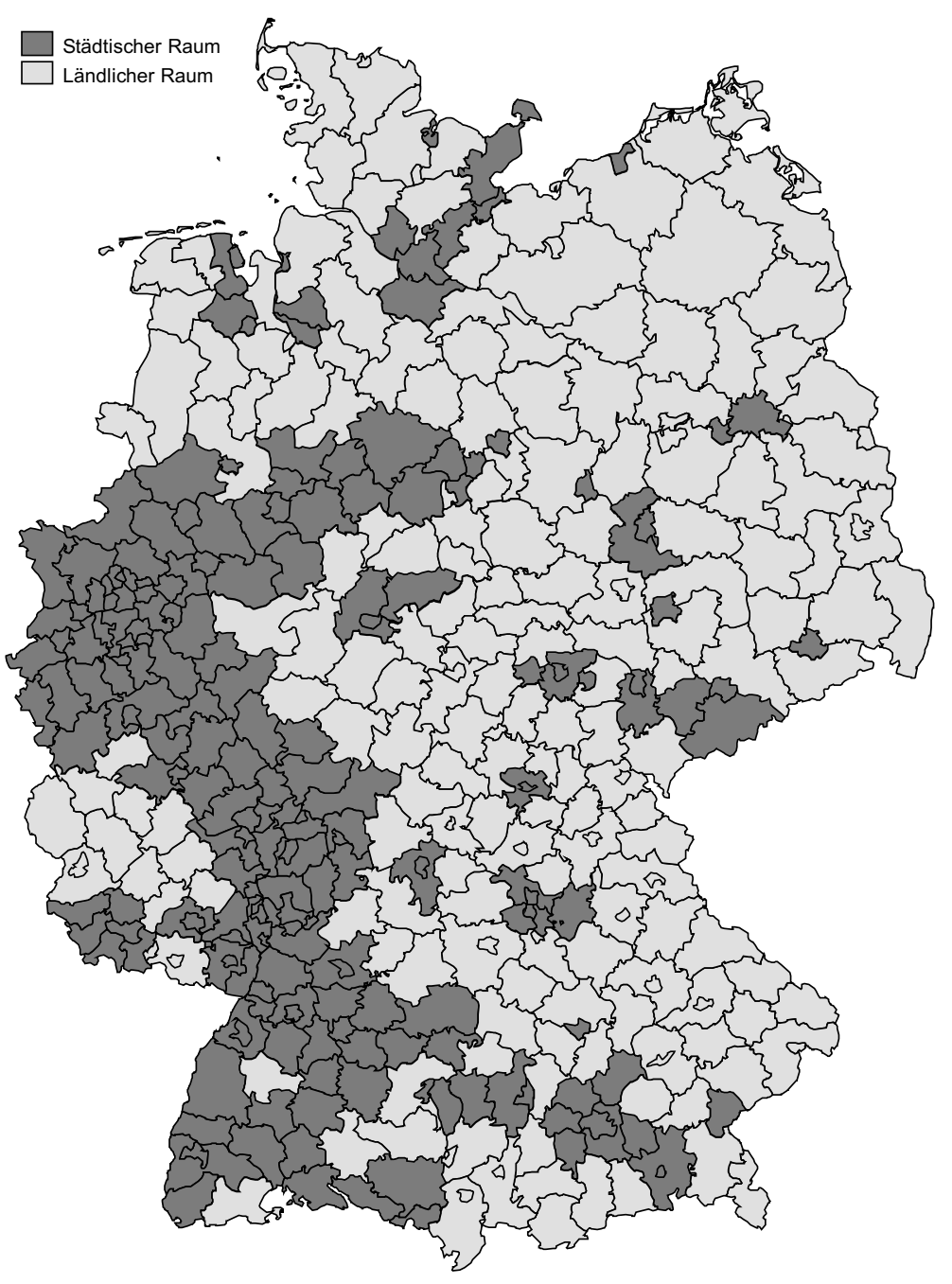

Quelle: INKAR-Datenbank des BBSR, Gebietsstand 31.12.2012; (c) GeoBasis-DE. Eigene Darstellung (DZA).

Stadt und Land: Der Regionstyp gibt wieder, ob es sich bei einem Landkreis oder einer kreisfreien Stadt um einen ländlichen oder städtischen Raum handelt. Alle kreisfreien Großstädte sowie die städtischen Kreise bilden den städtischen
Raum, alle ländlichen Kreise den ländlichen Raum (Bundesinstitut für Bau-, Stadt- und Raumforschung (BBSR) 2015c). Abbildung 21-1 zeigt die Verteilung der Regionstypen Stadt und Land in Deutschland. 


\section{Abbildung 21-2: Verteilung der vier siedlungsstrukturellen Kreistypen in Deutschland}

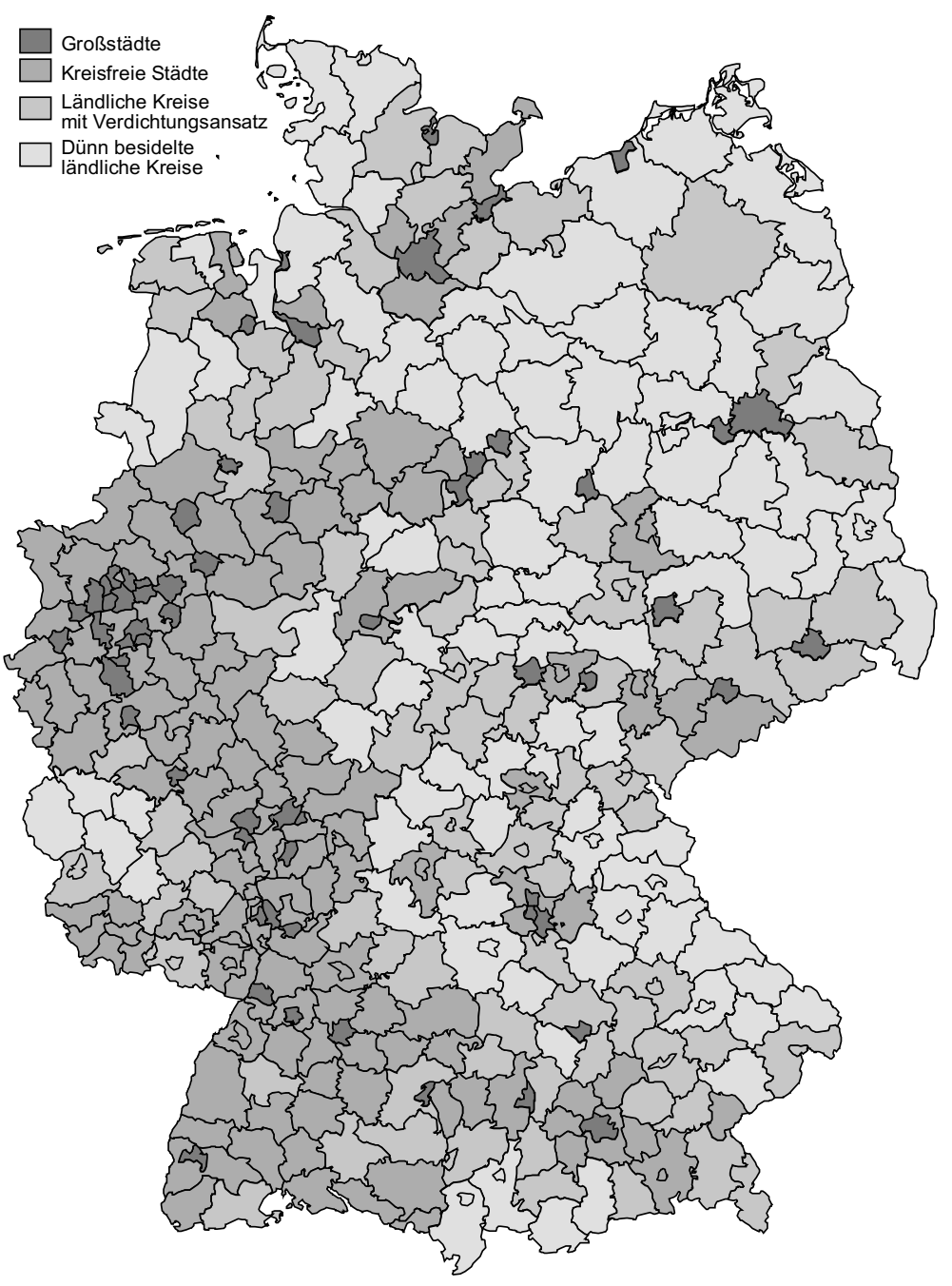

Quelle: INKAR-Datenbank des BBSR, Gebietsstand 31.12.2012; @ GeoBasis-DE. Eigene Darstellung (DZA).

Siedlungsstruktureller Kreistyp: Der Indikator ,siedlungsstruktureller Kreistyp ' differenziert den dichotomen Regionstyp ,Stadt und Land' in insgesamt vier Kategorien (Bundesinstitut für Bau-, Stadt- und Raumforschung (BBSR) 2015b). Abbildung 21-2 zeigt die Verteilung der vier siedlungsstrukturellen Kreistypen in Deutschland.
Die vier siedlungsstrukturellen Kreistypen werden wie folgt definiert:

- Kreisfreie Großstädte: Kreisfreie Städte mit mind. 100.000 Einwohnerinnen und Einwohnern 
- Städtische Kreise: Kreise mit einem Bevölkerungsanteil in Groß- und Mittelstädten von mindestens 50 Prozent und einer Einwohnerdichte von mind. 150 Einwohnerinnen und Einwohnern $/ \mathrm{km}^{2}$; sowie Kreise mit einer Einwohnerdichte ohne Groß- und Mittelstädte von mind. 150 Einwohnerinnen und Einwohnern $/ \mathrm{km}^{2}$

- Ländliche Kreise mit Verdichtungsansätzen: Kreise mit einem Bevölkerungsanteil in Großund Mittelstädten von mind. 50 Prozent, aber einer Einwohnerdichte unter 150 Einwohnerinnen und Einwohnern $/ \mathrm{km}^{2}$, sowie Kreise mit einem Bevölkerungsanteil in Groß- und Mittelstädten unter 50 Prozent mit einer Einwohnerdichte ohne Groß- und Mittelstädte von mind. 100 Einwohnerinnen und Einwohnern/ $\mathrm{km}^{2}$

- Dünn besiedelte ländliche Kreise: Kreise mit einem Bevölkerungsanteil in Groß- und Mittelstädten unter 50 Prozent und Einwohnerdichte ohne Groß- und Mittelstädte unter 100 Einwohnerinnen und Einwohnern $/ \mathrm{km}^{2}$

Arbeitslosenquote: Ein zentraler Indikator für die wirtschaftliche Prosperität einer Region ist die Arbeitslosenquote, ausgedrückt als Anteil der
Arbeitslosen an den zivilen Erwerbspersonen in Prozent (Bundesinstitut für Bau-, Stadt- und Raumforschung (BBSR) 2015a). Die durchschnittliche kreisspezifische Arbeitslosenquote beträgt 6,4 Prozent (damit entsprechen die Kreise Stade, Leer, Speyer, Schweinfurt (Stadt), Augsburg (Stadt) sowie Eichsfeld/Thüringen exakt dem Durchschnitt), sie reicht von 1,2 Prozent (im Kreis Eichstätt) bis 16,4 Prozent (im Kreis Uckermark). ${ }^{3}$ Für die Analysen wurden fünf Gruppen von Landkreisen und kreisfreien Städte gebildet, in denen jeweils 20 Prozent aller Kreise enthalten sind (Quintile). In der Abbildung 21-3 sind diese fünf Gruppen dargestellt. Bei den Kreisen mit der hellsten Graustufe (erstes Quintil) handelt es sich um diejenigen 20 Prozent der Kreise, in denen der Anteil an Arbeitslosen am niedrigsten ist (Arbeitslosenquote 1,2 bis 3,6 Prozent). Je dunkler das Grau, desto höher ist die Arbeitslosenquote im jeweiligen Kreis. Die Kreise mit der dunkelsten Graustufe (fünftes Quintil) sind diejenigen 20 Prozent der Kreise, in denen der Anteil an Arbeitslosen am höchsten ist (Arbeitslosenquote 9,2 bis 16,4 Prozent).

3 Die hier verwendeten Indikatoren wurden der Datenbank Indikatoren und Karten zur Raum- und Stadtentwicklung (INKAR) des Bundesinstituts für Bau-, Stadt- und Raumforschung (BBSR) entnommen (www.inkar.de). 


\section{Abbildung 21-3: Arbeitslosenquote, auf Kreisebene, in Quintilen}

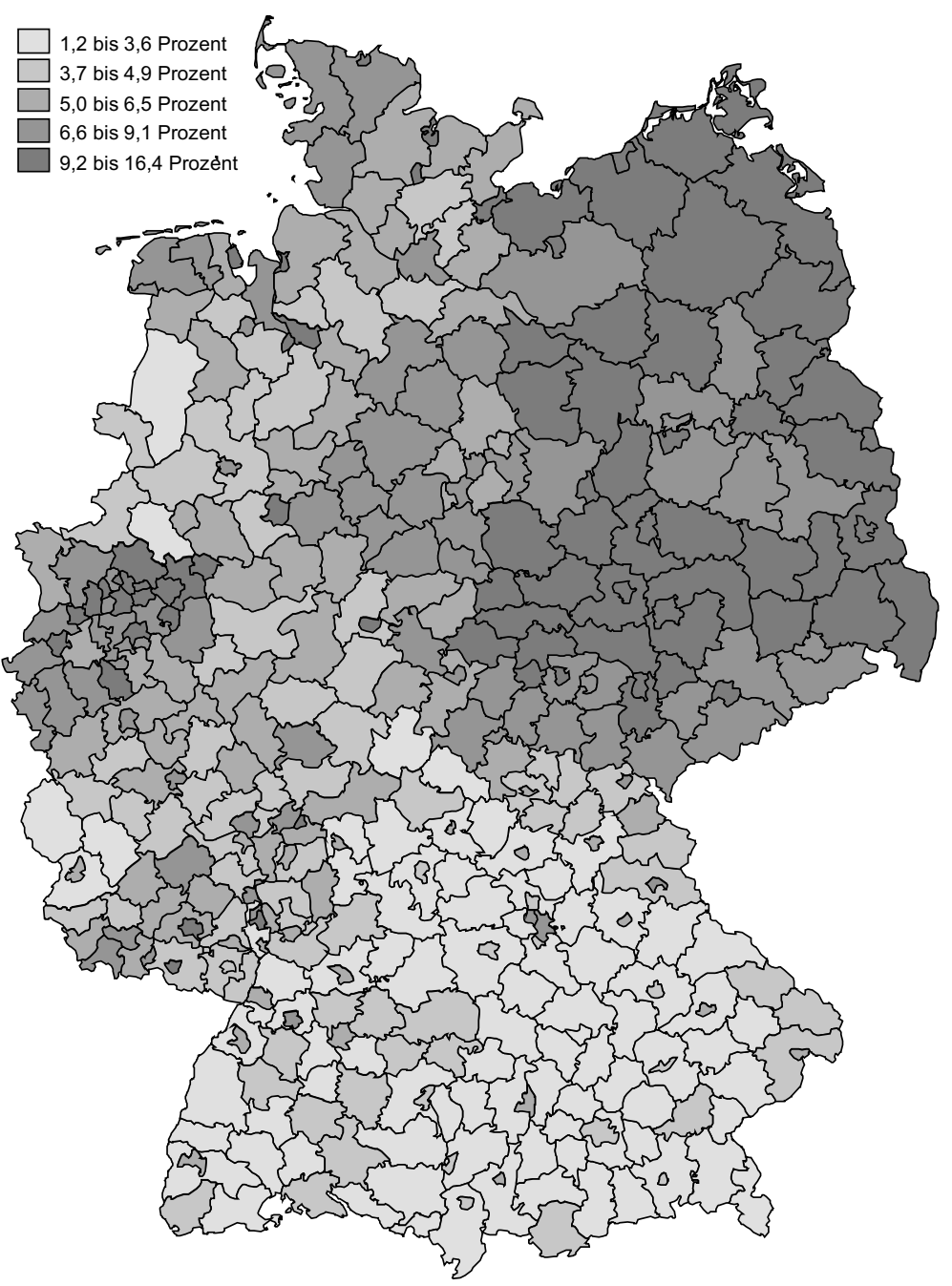

Quelle: INKAR-Datenbank des BBSR, Gebietsstand 31.12.2012; (C GeoBasis-DE. Eigene Berechnungen, eigene Darstellung (DZA). 


\subsection{Engagement auf dem Land und in der Stadt}

Wenn wir die Regionaltypen ,Stadt und Land" betrachten, stellen wir fest, dass die Engagementquote im ländlichen Raum mit 45,5 Prozent deutlich höher liegt als im städtischen Raum mit 42,7 Prozent (Abbildung 21-4). In ländlichen
Regionen gibt es möglicherweise einen größeren Bedarf, eine stärkere Förderung oder eine höhere Zahl von Gelegenheiten, sich freiwillig neben Beruf und Familie zu engagieren, als dies in städtischen Regionen der Fall ist.

Abbildung 21-4: Anteile freiwillig Engagierter nach Regionstyp ,Stadt und Land' 2014

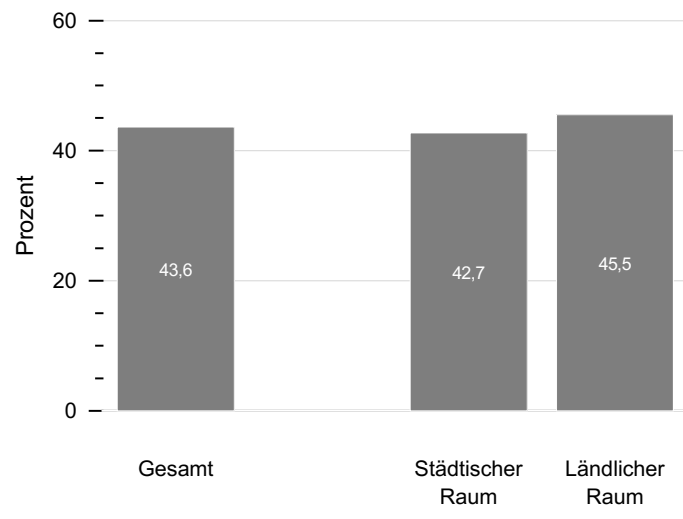

Quelle: FWS 2014, gewichtet, eigene Berechnungen (DZA). Basis: Alle Befragten mit gültigen Informationen zu Regionaldaten $(n=25.475)$.

Differenziert man innerhalb der Regionstypen ,Stadt und Land' nach Geschlecht, so zeigt sich, dass es in ländlichen Kreisen einen größeren Unterschied zwischen den Engagementquoten von Männern und Frauen gibt als in Städten (Abbildung 21-5a). Während im städtischen Raum der Abstand zwischen den Geschlechtern 2,7 Prozentpunkte beträgt (Frauen: 41,4 Prozent, Männer: 44,1 Prozent), liegt die Geschlechterdifferenz in ländlichen Gebieten bei 7,0 Prozentpunkten (Frauen: 42,0 Prozent; Männer:
49,0 Prozent). Für Frauen ist der Stadt-Land-Unterschied im freiwilligen Engagement nur klein (Frauen in der Stadt: 41,4 Prozent, Frauen auf dem Land: 42,0 Prozent), für Männer fällt er deutlich größer aus (Männer in der Stadt: 44,1 Prozent, Männer auf dem Land: 49,0 Prozent). Während sich die engagementfördernden Gegebenheiten für Frauen in Stadt und Land offensichtlich ähneln, sind Männer auf dem Land deutlich stärker in freiwillige Tätigkeiten eingebunden als in der Stadt. 
Abbildung 21-5: Anteile freiwillig Engagierter nach Regionstyp ,Stadt und Land' 2014, a) nach Geschlecht, b) nach Alter und c) nach Bildung

a) nach Geschlecht

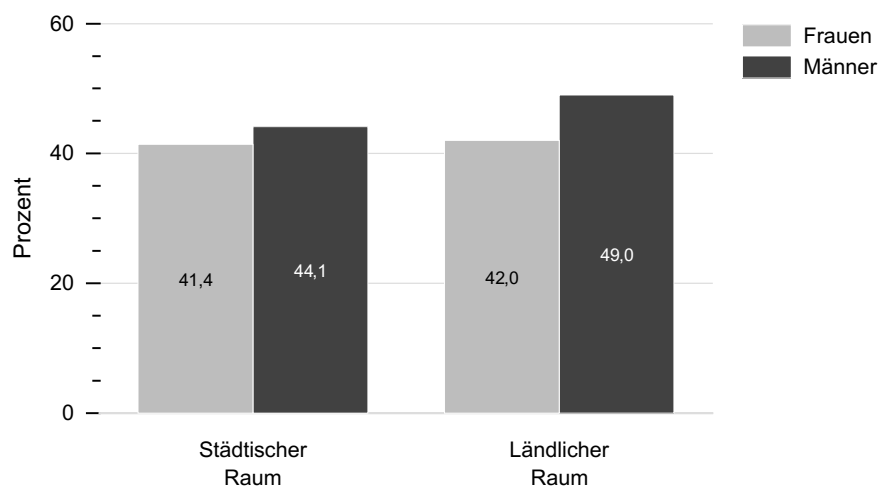

b) nach Alter

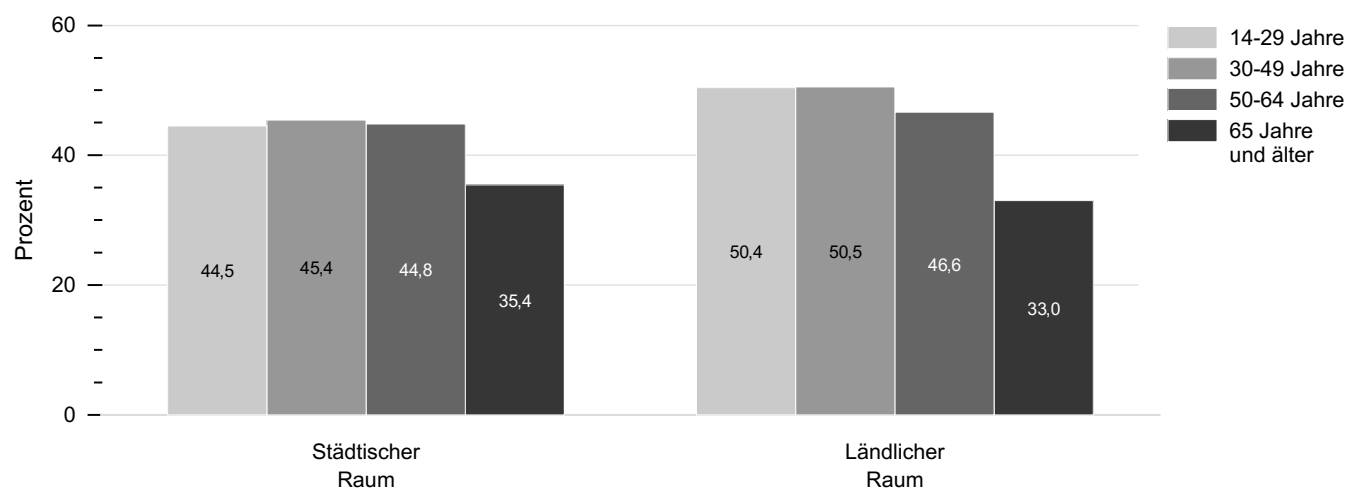

c) nach Bildung

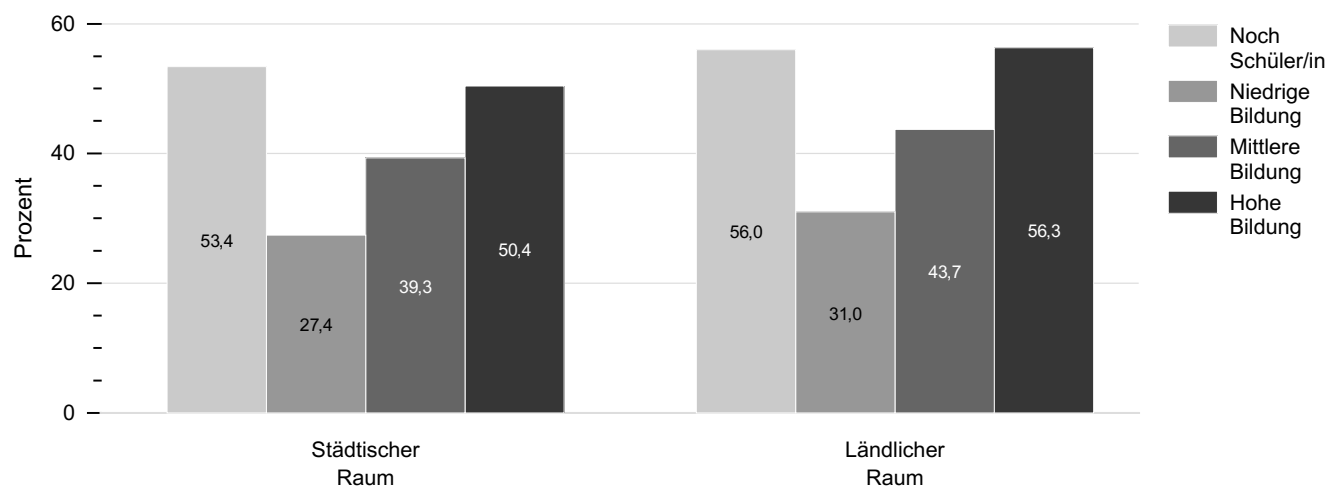

Quelle: FWS 2014, gewichtet, eigene Berechnungen (DZA). Basis: Alle Befragten mit gültigen Informationen zu Regionaldaten. Geschlecht und Alter $(n=25.475)$, Bildung $(n=25.468)$. 
Der positive Zusammenhang zwischen ländlichem Raum und der Beteiligung am freiwilligen Engagement zeigt sich auch bei der Differenzierung nach Altersgruppen (Abbildung 21-5b). Der Anteil Engagierter ist in den ersten drei Altersgruppen auf dem Land größer als in der Stadt. Nur in der ältesten Gruppe dreht sich dieser $\mathrm{Zu}$ sammenhang um. Bei Menschen, die 65 Jahre und älter sind, liegt der Anteil freiwillig Tätiger im ländlichen Raum mit 33,0 Prozent niedriger als im städtischen mit 35,4 Prozent. Dies lässt vermuten, dass die für das freiwillige Engagement förderlichen Strukturen für Ältere in städtischen Gebieten besser sind als für ältere Menschen, die in ländlichen Regionen leben. Möglicherweise ist die Angebotsstruktur auf dem Land stärker auf jüngere Personen ausgerichtet. Möglicherweise lassen sich diese Befunde aber auch auf Einschränkungen der Mobilität bei den 65-Jährigen und Älteren im ländlichen Raum zurückführen, etwa durch eine unzureichende Infrastruktur des öffentlichen Nahverkehrs.

Im Vergleich der Bildungsgruppen zeigen sich in allen vier Kategorien nahezu die gleichen Muster (Abbildung 21-5c). Die Differenzen zwischen den Bildungsgruppen sind sowohl in städtischen wie in ländlichen Regionen sehr ähnlich. Die Bedeutung des Bildungsstandes für freiwilliges Engagement ist im ländlichen und städtischen Raum also ähnlich groß. 


\subsection{Engagement in unterschiedlichen siedlungsstrukturellen Kreistypen}

Mithilfe des siedlungsstrukturellen Kreistyps können räumliche Unterschiede im freiwilligen Engagement differenzierter betrachtet werden: Hier unterscheiden wir zwischen vier Abstufungen der Verstädterung beziehungsweise Ländlichkeit: Kreisfreie Großstädte, städtische Kreise (diesen beiden Kreistypen bilden den Regionstyp ,städtischer Raum'), ländliche Kreise mit Verdichtungsansätzen und dünn besiedelte ländliche Kreise (diese beiden Kreistypen bilden den Regionstyp ,ländlicher Raum').

Es gibt einen deutlichen Unterschied in der Engagementquote zwischen den kreisfreien Großstädten mit 39,0 Prozent einerseits und den drei anderen Siedlungsstrukturtypen andererseits (Abbildung 21-6): In städtischen Kreisen liegt die Engagementquote bei 45,4 Prozent, in ländlichen Kreisen mit Verdichtungsansätzen bei 46,0 Prozent und in dünn besiedelten ländlichen Kreisen bei 45,0 Prozent. Die statistische Prüfung ergibt, dass sich die kreisfreien Großstädte signifikant von den drei anderen siedlungsstrukturellen Kreistypen unterscheiden. Die Unterschiede zwischen städtischen Kreisen, ländlichen Kreisen mit Verdichtungsansätzen und dünn besiedelten ländlichen Kreisen sind statistisch nicht bedeutsam.

\section{Abbildung 21-6: Anteile freiwillig Engagierter nach siedlungsstrukturellem Kreistyp 2014}

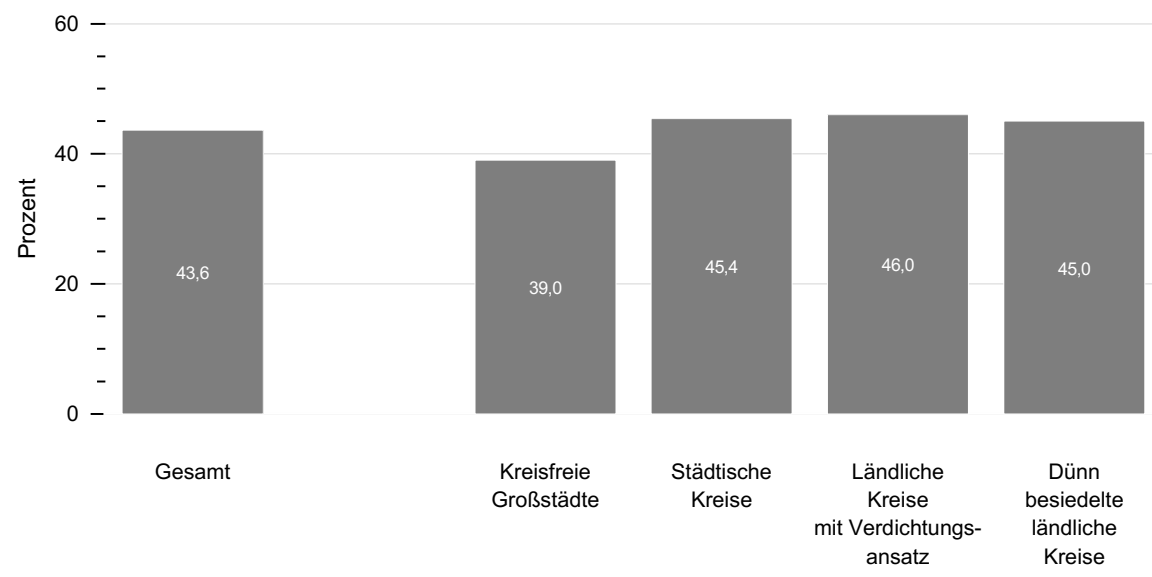

Quelle: FWS 2014, gewichtet, eigene Berechnungen (DZA). Basis: Alle Befragten mit gültigen Angaben zu Regionalinformationen $(n=25.475)$. 
In allen siedlungsstrukturellen Kreistypen fällt der Anteil Engagierter unter den Frauen signifikant niedriger aus als unter den Männern, aber in den kreisfreien Großstädten und den städtischen Kreisen sind die Geschlechterunterschiede etwas geringer als in den ländlichen Kreisen mit Verdichtungsansätzen und den dünn besiedelten ländlichen Kreisen (Abbildung 21-7a). Hervorzuheben ist auch, dass der höchste Anteil von Frauen, die sich freiwillig engagieren, in städtischen Kreisen zu finden ist. Möglicherweise ist in diesem Regionstypus die Engagementinfrastruktur am besten auf die Interessen von Frauen ausgerichtet.

Abbildung 21-7: Anteile freiwillig Engagierter nach siedlungsstrukturellem Kreistyp 2014, a) nach Geschlecht, b) nach Alter und c) nach Bildung

a) nach Geschlecht

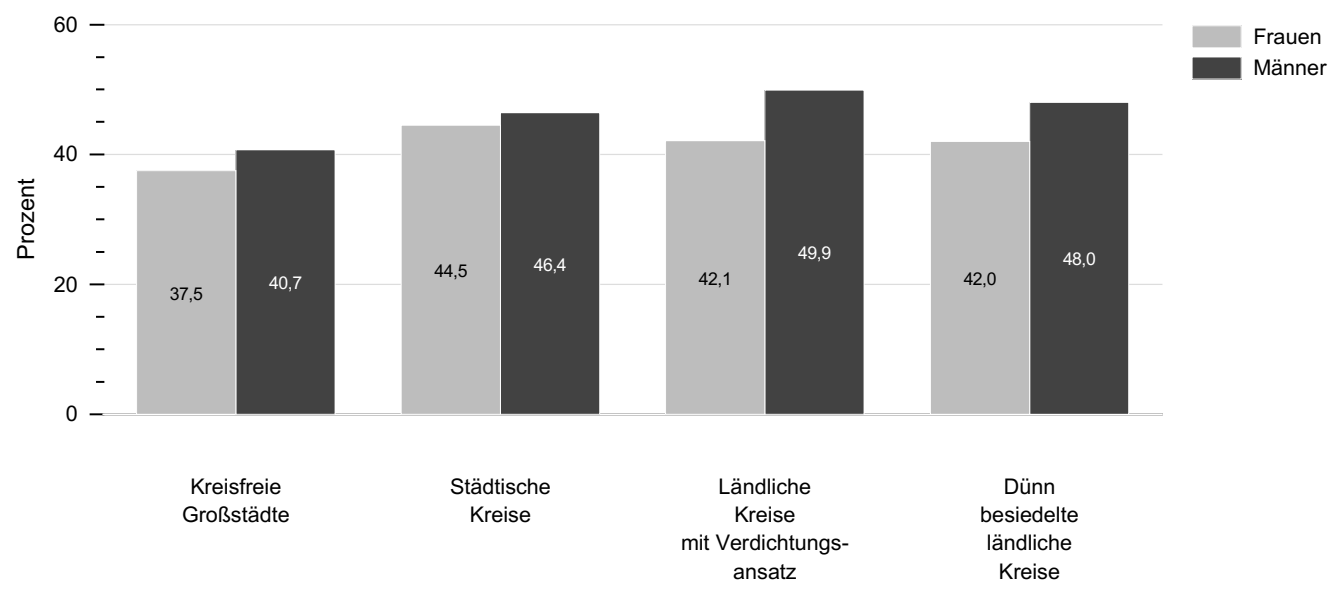

b) nach Alter

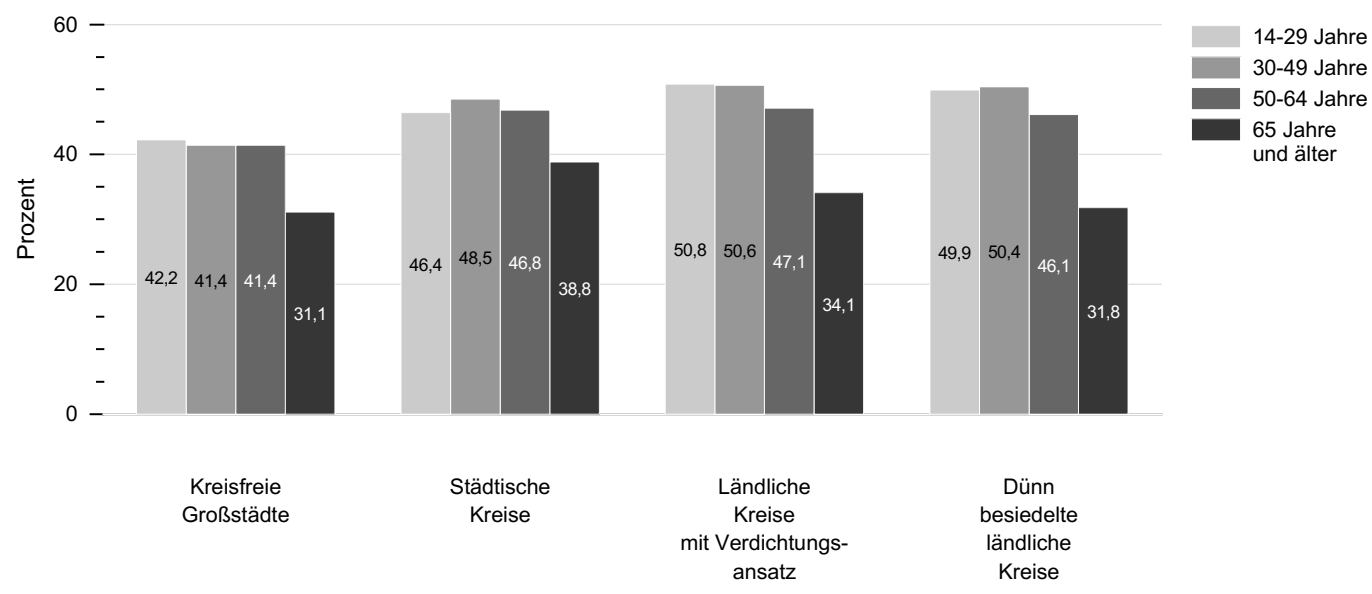


c) nach Bildung

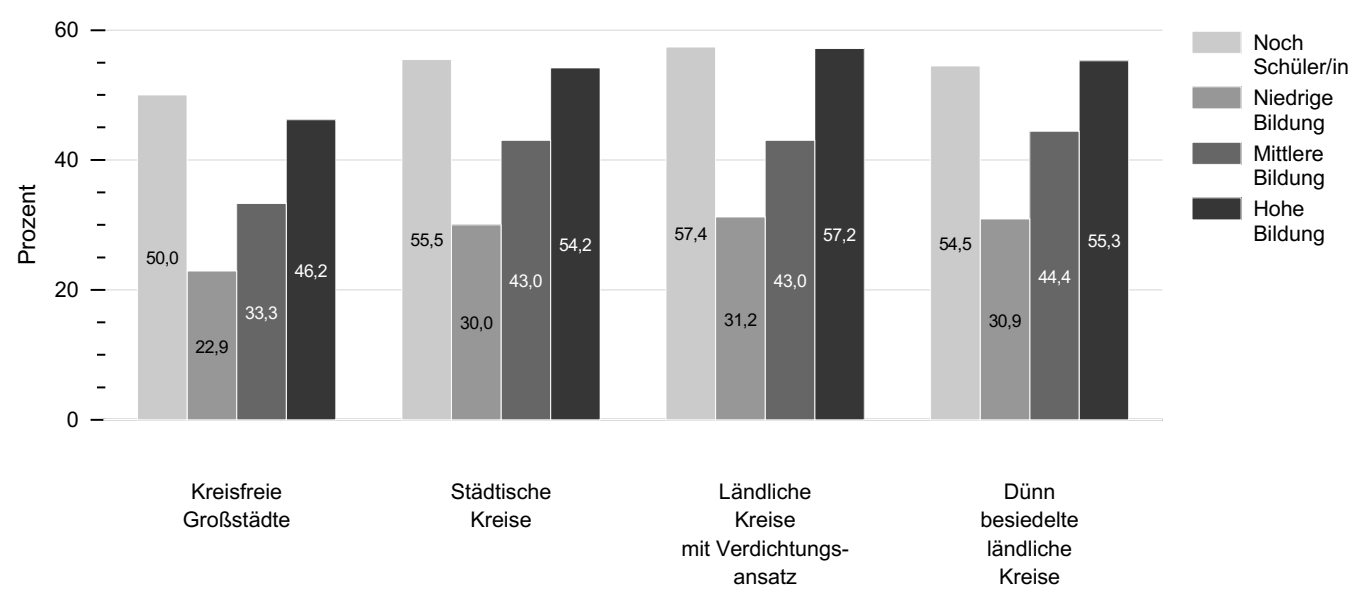

Quelle: FWS 2014, gewichtet, eigene Berechnungen (DZA). Basis: Alle Befragten mit gültigen Informationen zu Regionaldaten, Geschlecht und Alter $(n=25.475)$, Bildung $(n=25.468)$.

Über die siedlungsstrukturellen Kreistypen hinweg zeigen sich für die Altersgruppen ähnliche Ergebnismuster (Abbildung 21-7b): Die jüngeren Altersgruppen sind zu höheren Anteilen freiwillig engagiert als die älteren. Allerdings sind die Altersgruppenunterschiede in den beiden ländlichen Regionstypen stärker ausgeprägt als in den beiden städtischen Regionstypen. Mit Blick auf die älteste Altersgruppe (65 Jahre und älter) fällt insbesondere der Regionstyp der städtischen Kreise ins Auge: Dort ist die Engagementquote der ältesten Altersgruppe mit 38,8 Prozent deut- lich höher als in den drei anderen siedlungsstrukturellen Kreistypen.

Die Verteilung der Bildungsgruppen ist in allen vier siedlungsstrukturellen Kreistypen sehr ähnlich: Die niedrigste Engagementquote findet sich durchgehend bei den Personen mit niedriger Bildung, die höchste bei den Personen mit hoher Bildung beziehungsweise den Schülerinnen und Schülern (Abbildung 21-7c). Der Bildungsstand einer Person ist also in allen räumlichen Kontexten von hoher Bedeutung. 


\subsection{Engagement in Regionen mit unterschiedlichem Niveau der Arbeitslosigkeit}

\begin{abstract}
Abschließend untersuchen wir den Zusammenhang zwischen der wirtschaftlichen Lage in einer Region und dem Anteil von Menschen, die sich in dieser Region freiwillig engagieren. Die Arbeitslosenquote ist ein Indikator für die wirtschaftliche Lage eines Kreises. In den folgenden Analysen wird untersucht, ob die Arbeitslosenquote einer Region mit dem Engagement ihrer Einwohnerinnen und Einwohner zusammenhängt. Für die Analysen wurden fünf Gruppen von Landkreisen und kreisfreien Städte gebildet, in denen jeweils 20 Prozent aller Kreise enthalten sind (Quintile). Die Gruppe mit der Bezeichnung,erstes Quintil‘ enthält diejenigen 20 Prozent der Landkreise und kreisfreien Städte, in denen der Anteil an Arbeitslosen am niedrigsten ist (Arbeitslosenquote 1,2 bis 3,6 Prozent). Die Gruppe mit der Bezeichnung ,fünftes Quintil' enthält diejenigen 20 Prozent der Kreise, in denen der Anteil an Arbeitslosen am höchsten ist (Arbeitslosenquote 9,1 bis 16,4 Prozent).

Abbildung 21-8 zeigt die Engagementquoten in den Gruppen von Landkreisen und kreisfreien
\end{abstract}

Städten mit unterschiedlicher Arbeitslosenquote. Regionen mit geringer Arbeitslosigkeit weisen eine höhere Engagementquote auf als Regionen mit hoher Arbeitslosigkeit. In der Gruppe der Landkreise und kreisfreien Städten mit der niedrigsten Arbeitslosigkeit (erstes Quintil: Arbeitslosenquote zwischen 1,2 Prozent und 3,6 Prozent) sind 49,5 Prozent der Einwohnerinnen und Einwohner freiwillig engagiert, in der Gruppe der Landkreise und kreisfreien Städten mit der höchsten Arbeitslosigkeit (fünftes Quintil: Arbeitslosenquote zwischen 9,1 Prozent und 16,4 Prozent) sind dagegen nur 37,9 Prozent der Einwohnerinnen und Einwohner freiwillig engagiert. Nur die Gruppe mit der niedrigsten Arbeitslosigkeit (erstes Quintil) unterscheidet sich statistisch bedeutsam von den vier anderen Gruppen. Die vier anderen Gruppen (zweites bis fünftes Quintil) unterscheiden sich nicht signifikant voneinander. Mit Blick auf freiwilliges Engagement ist also eine sehr niedrige Arbeitslosenquote förderlich für freiwilliges Engagement. 
Abbildung 21-8: Anteile freiwillig Engagierter in Regionen mit unterschiedlicher Arbeitslosenquote 2014

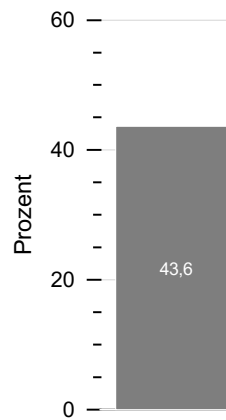

Gesamt

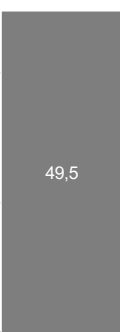

1. Quintil

AL-Quote $1,2-3,6 \%$

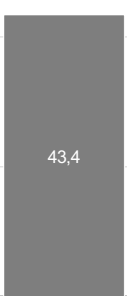
2. Quintil
AL-Quote 3,7-4,9\%

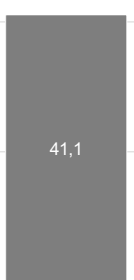
3. Quintil
AL-Quote 5,0-6,5\%

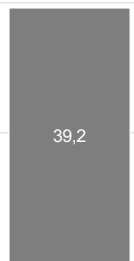

4. Quintil

AL-Quote 6,6-9,1\%

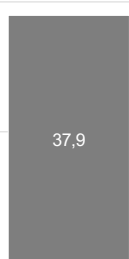

5. Quintil

AL-Quote 9,2-16,4\%

Quelle: FWS 2014, gewichtet, eigene Berechnungen (DZA). Basis: Alle Befragten mit gültigen Informationen zu Regionaldaten $(n=25.475)$. AL-Quote: Arbeitslosenquote. 
Über die Gruppen von Landkreisen und kreisfreien Städten mit unterschiedlich hoher Arbeitslosenquote zeigt sich ein sehr ähnliches Muster für Frauen und Männer, Menschen unterschiedlichen Alters und Personen unterschiedlicher Bildung. Unabhängig von der wirtschaftlichen Lage der Landkreise und kreisfreien Städten zeigt sich bei Männern ein höherer Anteil freiwillig Enga- gierter als bei Frauen (Abbildung 21-9a). Dies gilt auch für Altersgruppen: Altersunterschiede im freiwilligen Engagement sind über Regionen mit unterschiedlicher Arbeitslosigkeit sehr ähnlich (Abbildung 21-9b). Auch für Bildungsunterschiede ist ein recht ähnliches Muster über die Regionen mit unterschiedlich hoher Arbeitslosigkeit zu konstatieren (Abbildung 21-9c).

Abbildung 21-9: Anteile freiwillig Engagierter in Regionen mit unterschiedlicher Arbeitslosenquote 2014, a) nach Geschlecht, b) nach Alter und c) nach Bildung

a) nach Geschlecht

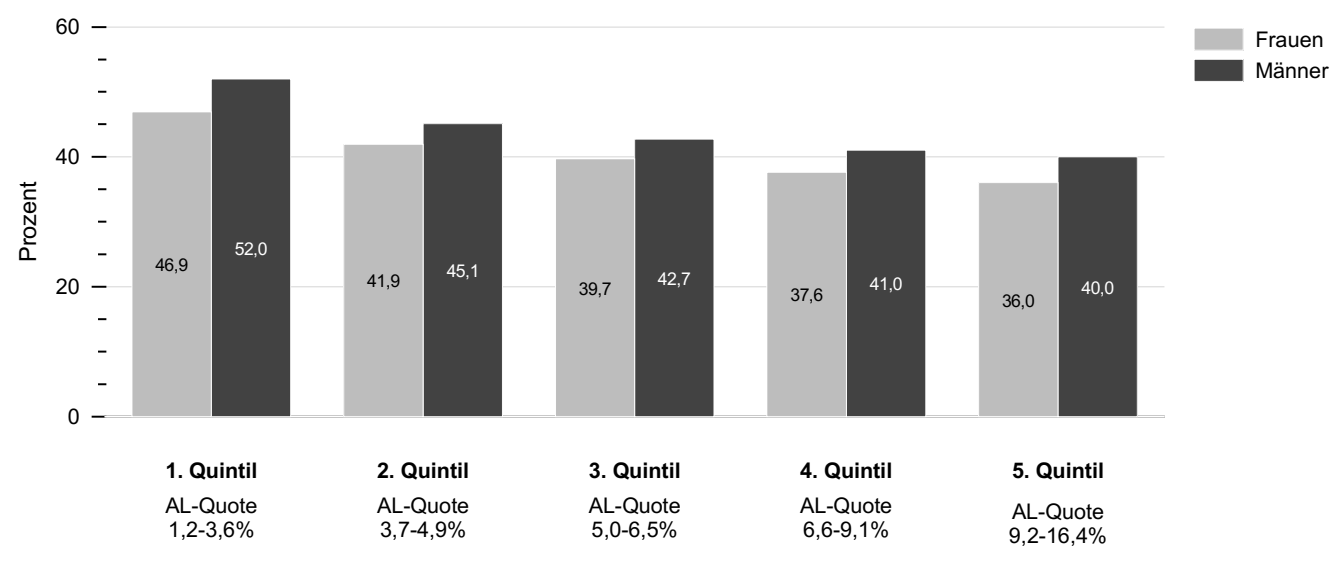

b) nach Alter

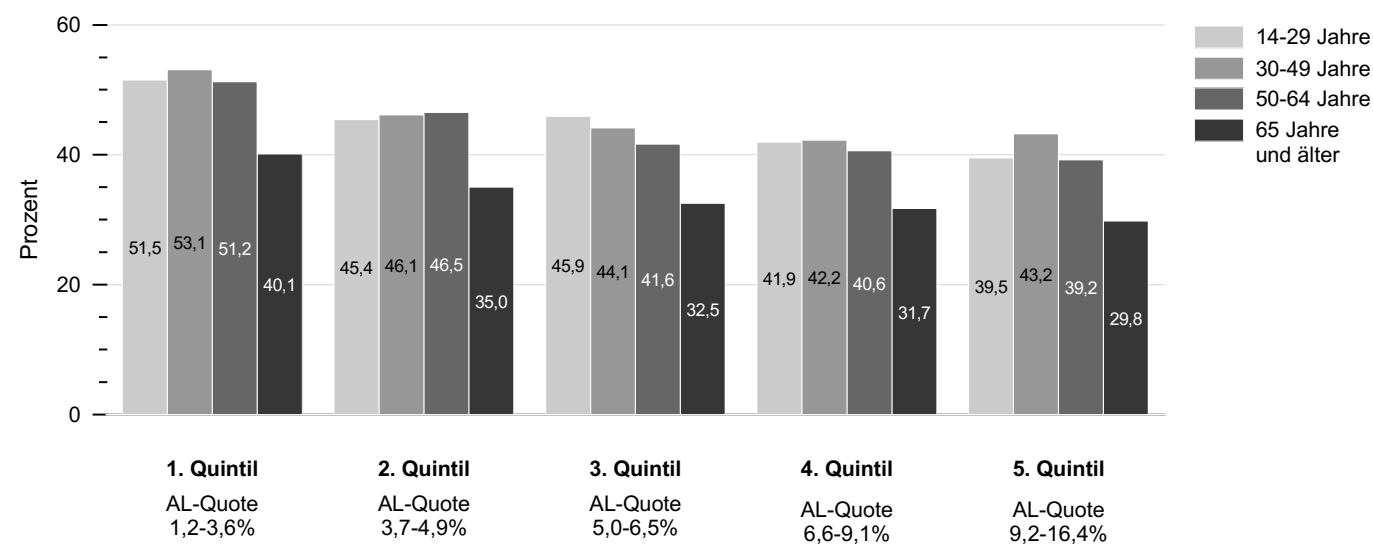


c) nach Bildung

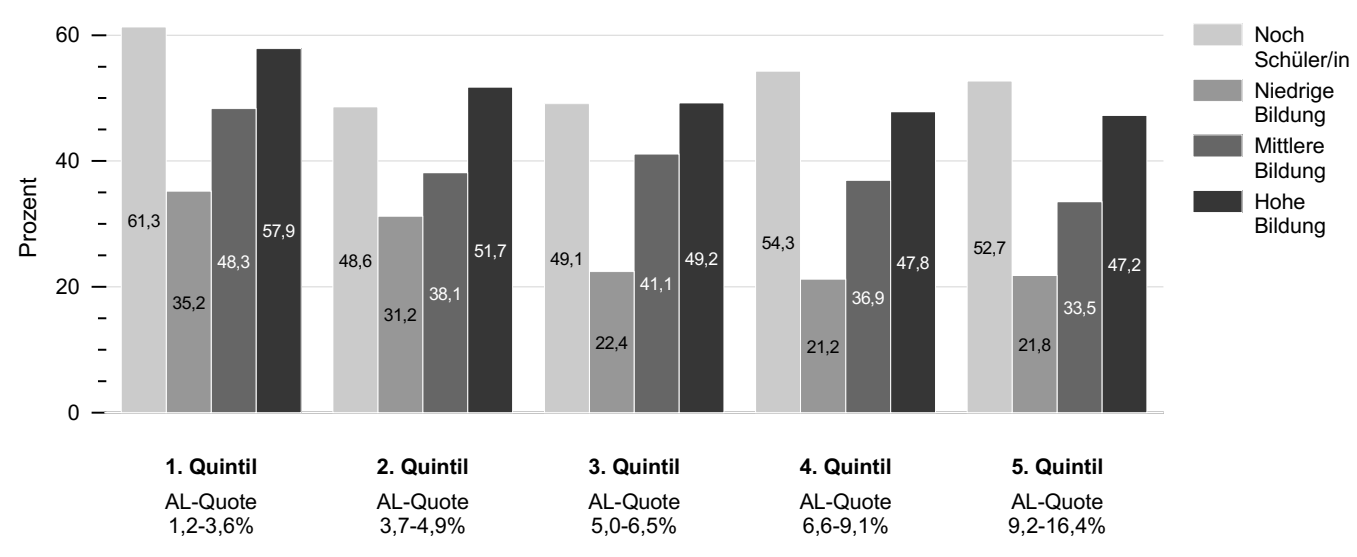

Quelle: FWS 2014, gewichtet, eigene Berechnungen (DZA). Basis: Alle Befragten mit gültigen Informationen zu Regionaldaten. Geschlecht und Alter $(n=25.475)$, Bildung $(n=25.468)$. AL-Quote: Arbeitslosenquote. 


\subsection{Freiwilliges Engagement: Individuelle Merkmale und regionaler Kontext}

Die bisherigen Analysen zeigen eindrucksvoll, dass es regionale Unterschiede im freiwilligen Engagement gibt. In ländlichen Regionen ist der Anteil freiwillig engagierter Menschen höher als in städtischen Regionen, und in wirtschaftlich starken Regionen mit geringer Arbeitslosigkeit ist der Anteil freiwillig engagierter Menschen höher als in wirtschaftlich schwächeren Regionen mit hoher Arbeitslosigkeit. Allerdings stellt sich nun die Frage, die wir bereits zu Beginn dieses Kapitels angesprochen haben: Handelt es sich bei diesen Unterschieden um Kontexteffekte oder um Kompositionseffekte? Unterschiedliche Regionstypen unterscheiden sich nicht allein mit Blick auf Siedlungsweise, Infrastruktur und Wirtschaftskraft, sondern auch mit Blick auf die Zusammensetzung der Bevölkerung. So ist die Bevölkerung auf dem Land anders zusammengesetzt als in der Stadt. Menschen mit hoher Bildung sind beispielsweise zu einem größeren Anteil in Städten als auf dem Land zu finden. Bei der Analyse räumlicher Kontexte darf nicht vergessen werden, dass viele individuelle Merkmale, wie Geschlecht, Alter, Bildung und Einkommen mit der Wahrscheinlichkeit zusammenhängen, sich freiwillig zu engagieren (siehe Kapitel 16). Unterschiede zwischen unterschiedlichen Regionstypen hinsichtlich des Anteils freiwillig engagierter Menschen könnten also auch auf die Zusammensetzung der Bevölkerung in diesen Regionen zurückgeführt werden.

Um diese Überlegungen in einem Analysemodell zu überprüfen, wählen wir eine logistische Mehrebenen-Regression mit Engagement (ja/nein) als abhängiger Variable. Mit Hilfe dieser Methode können wir Unterschiede im Engagement, die auf den Merkmalen von Kreisen beruhen, trennen von Zusammenhängen, die von individuellen Charakteristika der befragten Personen herrühren. In der von uns durchgeführten Mehrebenen-Regressionen unterschei- den wir zwei Ebenen: Die erste Ebene umfasst Individuen, die zweite Ebene räumliche Einheiten. In unserer Analyse wird der Einfluss von Merkmalen auf der individuellen Ebene statistisch kontrolliert. Diese individuellen Merkmale sind Geschlecht, Alter, Bildung, subjektives Einkommen, subjektive Gesundheit, Werthaltung Solidarität sowie die Design-Variable ,Kontakt über Festnetz/Mobilfunk'. Auf der zweiten Analyseebene wird der Einfluss der Kreiszugehörigkeit untersucht. Wir charakterisieren die Kreise mit drei Fokusvariablen (Regionstyp ,Stadt und Land', siedlungsstruktureller Kreistyp sowie Regionen mit unterschiedlicher Arbeitslosenquote). Wenn für die individuellen Merkmale statistisch kontrolliert wird, zeigen die Koeffizienten der zweiten Ebene die reinen Effekte des räumlichen Kontextes. Wir berichten unstandardisierte Koeffizienten. Positive Koeffizienten geben an, dass eine positive Beziehung vorliegt, die Wahrscheinlichkeit sich zu engagieren also steigt, negative Koeffizienten stehen für eine negative Beziehung. Dargestellt sind nur statistisch signifikante Koeffizienten, nicht signifikante Koeffizienten werden durch drei Striche angezeigt.

Eine statistische Maßzahl, der Intra-Klassen-Koeffizient, gibt an, welcher Anteil der Varianz des freiwilligen Engagements auf Unterschiede zwischen räumlichen Einheiten und welcher Anteil auf Unterschieden zwischen Personen zurückzuführen ist. Ein hoher Intra-Klassen-Koeffizient zeigt an, dass die Varianz des freiwilligen Engagements in hohem Maße von den räumlichen Kontexten beeinflusst wird. In Tabelle 21-1 ist ersichtlich, dass der Intra-Klassen-Koeffizient recht niedrig ist (Regionstyp ,Stadt und Land': 0,017, siedlungsstruktureller Kreistyp: 0,013, Regionen mit unterschiedlicher Arbeitslosenquote: 0,009). Die individuellen Merkmale der Befragten sind bei der Vorhersage des freiwilligen Engage- 
ments also von sehr viel stärkerer Bedeutung als die räumlichen Kontexte. Allerdings zeigt sich bei den Koeffizienten der logistischen Mehr-
ebenen-Regression, dass auch die räumlichen Kontexte von Bedeutung sind.

Tabelle 21-1: Ergebnisse logistischer Mehrebenen-Regressionen. Vorhersage freiwilligen Engagements durch drei Fokusvariablen: Regionstyp ,Stadt und Land', siedlungsstruktureller Kreistyp sowie Regionen mit unterschiedlicher Arbeitslosenquote, 2014

\begin{tabular}{|c|c|c|c|}
\hline & $\begin{array}{c}\text { Regionstyp } \\
\text {,Stadt und Land' }\end{array}$ & $\begin{array}{c}\text { Siedlungsstruktureller } \\
\text { Kreistyp }\end{array}$ & $\begin{array}{c}\text { Regionen mit } \\
\text { unterschiedlicher } \\
\text { Arbeitslosenquote }\end{array}$ \\
\hline \multicolumn{4}{|c|}{ Regionstyp ,Stadt und Land' (Referenz: Ländlicher Raum) } \\
\hline Städtischer Raum & $-0,13^{* *}$ & & \\
\hline \multicolumn{4}{|c|}{ Siedlungsstruktureller Kreistyp (Referenz: Ländliche Kreise mit Verdichtungsansatz) } \\
\hline Kreisfreie Großstädte & & $-0,30 * * *$ & \\
\hline Städtische Kreise & & --- & \\
\hline Dünn besiedelte ländliche Kreise & & --- & \\
\hline \multicolumn{4}{|c|}{ Regionen unterschiedlicher Arbeitslosigkeit (Referenz: Zweites Quintil: 3,7-4,9\% AL-Quote) } \\
\hline Erstes Quintil: 1,2-3,6\% AL-Quote & & & $0,25 * * *$ \\
\hline Drittes Quintil: 5,0-6,5\% AL-Quote & & & --- \\
\hline Viertes Quintil: 6,6-9,1 \% AL-Quote & & & $-0,20 * *$ \\
\hline Fünftes Quintil: 9,2-16,4 \% AL-Quote & & & $-0,29 * * *$ \\
\hline Pseudo R2 & 0,07 & 0,07 & 0,07 \\
\hline Intra-Klassen-Korrelationskoeffizient Rho & 0,017 & 0,013 & 0,009 \\
\hline Anzahl gültiger Fälle & 24.889 & 24.889 & 24.889 \\
\hline
\end{tabular}

Quelle: FWS 2014, ungewichtet, eigene Berechnungen (DZA). Kontrolliert für Geschlecht,

Alters- und Bildungsgruppe, subjektives Einkommen, subjektive Gesundheit, Werthaltung Solidarität sowie Design-Variable Kontakt über Festnetz/Mobilfunk). ${ }^{*} p<0,05,{ }^{* *} p<0,01, * * * p<0,001,--$ nicht signifikant.

Als Referenzkategorie haben wir jeweils folgende Kategorien gewählt: Regionstyp ,Stadt und Land‘: ländlicher Raum; siedlungsstruktureller Kreistyp: ländliche Kreise mit Verdichtungsansatz; Regionen mit unterschiedlicher Arbeitslosenquote: zweites Quintil mit einer Arbeitslosenquote zwischen 3,7 und 4,9 Prozent. Trotz der Einbeziehung von individuellen Merkmalen sind die regionalen Fokusvariablen statistisch bedeutsam. Die Analysen bestätigen also die Befunde aus den deskriptiven Darstellungen.
- Betrachtet man die Fokusvariable ,Regionstyp Stadt und Land', so sieht man, dass - im Vergleich zum ländlichen Raum - im städtischen Raum die Wahrscheinlichkeit, freiwillig engagiert zu sein, deutlich gesenkt ist (der Koeffizient beträgt -0,13).

- Betrachtet man die Fokusvariable ,Siedlungsstruktureller Kreistyp, so sieht man, dass - im Vergleich zu ländlichen Kreisen mit Verdichtungsansätzen - in Großstädten die Wahrscheinlichkeit, freiwillig engagiert $\mathrm{zu}$ sein, 
deutlich gesenkt ist (der Koeffizient beträgt $-0,30)$. Keine Unterschiede gibt es zwischen der Referenzkategorie und den städtischen Kreisen sowie den dünn besiedelten ländlichen Kreisen.

- Betrachtet man die Fokusvariable ,Regionen mit unterschiedlicher Arbeitslosigkeit', so sieht man, dass - im Vergleich zu den Regionen mit einer Arbeitslosenquote von 3,7 bis 4,9 Prozent - in den beiden Regionen mit den höchsten Ar- beitslosenquoten (Arbeitslosigkeit 6,6 Prozent und mehr) die Wahrscheinlichkeit für freiwilliges Engagement deutlich gesenkt ist (die Koeffizienten lauten $-0,20$ und -0,29). Dagegen ist in Regionen mit der niedrigsten Arbeitslosenquote (1,2 bis 3,6 Prozent) der Anteil von freiwillig Engagierten deutlich höher (der Koeffizient beträgt $+0,25$ ). Insgesamt gilt also: Je höher die allgemeine Arbeitslosigkeit in einer Region ist, desto niedriger ist die Wahrscheinlichkeit, sich freiwillig zu engagieren.

\subsection{Fazit}

Der Anteil freiwillig engagierter Menschen ist in Deutschland regional ungleich verteilt. Die Engagementquoten unterscheiden sich hinsichtlich Land und Stadt, zwischen unterschiedlichen siedlungsstrukturellen Kreistypen und zwischen Regionen mit unterschiedlich starkem Arbeitsmarkt. Diese Unterschiede zeigen sich auch dann, wenn man für die unterschiedliche Bevölkerungszusammensetzung in den regionalen Einheiten kontrolliert. Wir finden also Effekte des räumlichen Kontextes (nicht Effekte der Bevölkerungskomposition). Soziale Teilhabe im Sinne des Ausübens eines freiwilligen Engagements ist in Deutschland also nicht nur sozial ungleich verteilt, sondern ist auch von den regional-räumlichen Kontexten abhängig, in denen Menschen leben.

Am niedrigsten ist der Anteil freiwillig engagierter Menschen in Großstädten. Deutlich höher ist die Engagementquote in städtischen Kreisen, in ländlichen Kreisen mit Verdichtungsansätzen und in dünn besiedelten Landkreisen. Die Unterschiede lassen sich möglicherweise damit erklären, dass in der Stadt viele Angebote im Freizeitbereich mit jenen des freiwilligen Engagements konkurrieren, während in ländlichen Räumen Angebote des freiwilligen Engagements in stärkerem Maß der sozialen Integration dienen. Räumliche Kontexte scheinen sich auch unterschiedlich auf unterschiedliche Bevölkerungsgruppen auszuwirken. Ein Beispiel sind ältere Menschen: In der Altersgruppe der über 65-Jährigen ist die Beteiligung am Engagement besonders in den städtischen Kreisen sehr hoch. Möglicherweise sind in diesem Siedlungsstrukturtyp die Infrastrukturen für das Engagement älterer Menschen besonders günstig. Dieser Befund sollte bei der Planung von Fördermaßnahmen für freiwilliges Engagement berücksichtigt werden.

Aber auch die wirtschaftliche Prosperität einer Region ist wichtig für das freiwillige Engagement: Je stärker der Arbeitsmarkt in einer Region ist, desto höher ist die Engagementquote. In Regionen mit einer niedrigen Arbeitslosenquote ist der Anteil freiwillig Engagierter hoch. Dagegen ist in Regionen mit einer hohen Arbeitslosenquote der Anteil von freiwillig Engagierten deutlich niedriger. Welche Konsequenzen könnten diese Befunde für Maßnahmen der Engagementförderung haben? Möglicherweise lohnt es sich, vor allem jene Regionen in den Fokus zu nehmen, in denen die Engagementquote aufgrund ungünstiger wirtschaftlicher Rahmenbedingungen eher gering ist (vgl. Elbe \& Müller 2015; Staemmler 2015). Zugleich ist es plausibel anzunehmen, dass der Bedarf für freiwilliges Engagement in wirtschaftlich schwächeren Regionen besonders hoch ist. Allerdings bedarf es unter eher ungünstigen räumlichen Bedingungen besonders guter kommunaler Engagementförderung, um engage- 
mentbereite Menschen dabei zu unterstützen, sich freiwillig zu engagieren.

\section{Literatur}

Bundesinstitut für Bau-, Stadt- und Raumforschung (BBSR) (2015a). Indikatoren und Karten zur Raum- und Stadtentwicklung. INKAR. Ausgabe 2014. Bonn: BBSR.

Bundesinstitut für Bau-, Stadt- und Raumforschung (BBSR) (2015b). Laufende Raumbeobachtung - Raumabgrenzungen. Siedlungsstrukturelle Kreistypen. Online: http://www.bbsr.bund.de/BBSR/DE/Raumbeobachtung/Raumabgrenzungen/Kreistypen4/kreistypen.html?nn=443270 (zuletzt abgerufen 5.2.2016).

Bundesinstitut für Bau-, Stadt- und Raumforschung (BBSR) (2015c). Laufende Raumbeobachtung - Raumabgrenzungen. Städtischer und Ländlicher Raum. Online: http://www.bbsr.bund.de/BBSR/DE/Raumbeobachtung/Raumabgrenzungen/Kreistypen2/kreistypen.html?nn=443270 (zuletzt abgerufen 5.2.2016).

Bundesinstitut für Bau-, Stadt- und Raumforschung (BBSR) (2012). Raumordnungsbericht 2011. Bonn: BBSR.

Brauer, K. (2015). Bowling mit Wölfen: Rurale Gemeinden zwischen demographischem Untergang und (sozial-)kapitaler Zukunft. In: U. Fachinger \& H. Künemund (Hrsg.) Gerontologie und ländlicher Raum (S. 45-75). Wiesbaden: Springer VS.

Elbe, S., \& Müller, R. (2015). Gleichwertigkeit als Bürgeraufgabe. Partizipation der Zivilgesellschaft. In: H. Herrmann \& M. Eltges (Hrsg.) Gleichwertigkeit auf dem Prüfstand (S. 57-69). Stuttgart: Franz Steiner Verlag.

Gensicke, T. (2015). Freiwilliges Engagement in Deutschland: Freiwilligensurvey 2009. Wiesbaden: Springer VS.

Kawachi, I., \& Berkman, L. F. (Hrsg.) (2003). Neighborhoods and health. New York: Oxford University Press.

Neu, C. (2007). Bürgerschaftliches Engagement als Erfolgsfaktor für den ländlichen Raum. UNESCO heute, 54(2), 34-37.

Neu, C., \& Nikolic, L. (2014). Die (neuen) Selbstversorger - Zwischen Not und Weltanschauung? In: P. A. Berger, C. Keller, A. Klärner \& R. Neef (Hrsg.) Urbane Ungleichheiten. Neue Entwicklungen zwischen Zentrum und Peripherie (S. 253-271). Wiesbaden: Springer VS.

Scherger, S., Brauer, K., \& Künemund, H. (2004). Partizipation und Engagement älterer Menschen - Elemente der Lebensführung im Stadt-Land-Vergleich. In: G. M. Backes, W. Clemens \& H. Künemund (Hrsg.) Lebensformen und Lebensführung im Alter (S. 173-192). Wiesbaden: VS Verlag für Sozialwissenschaften.

Simonson, J., Hagen, C., Vogel, C., \& Motel-Klingebiel, A. (2013). Ungleichheit sozialer Teilhabe im Alter. Zeitschrift für Gerontologie und Geriatrie, 46(5), 410-416.

Staemmler, J. (2015). Städte auf der Suche nach sich selbst. Überlegungen zur Bedeutung der Zivilgesellschaft in strukturschwachen Kommunen. In: C. Eichert, R. Löffler \& Hohn, Stephanie (Hrsg.) Landflucht 3.0. (S. 52-65). Freiburg im Breisgau: Herder.

Strubelt, W. (1998). Stadt - Land. In: B. Schäfers \& W. Zapf (Hrsg.) Handwörterbuch zur Gesellschaft Deutschlands (S. 652-665). Bonn: Bundeszentrale für politische Bildung.

Wilson, J. (2012). Volunteerism Research: A Review Essay. Nonprofit and Voluntary Sector Quarterly, 41(2), 176-211.

Wilson, J. (2000). Volunteering. Annual Reviews in Sociology, 26, 215-240.

Zimmer, A., \& Backhaus-Maul, H. (2012). Engagementförderung vor Ort - Was gilt es in den Blick zu nehmen? Eine Arbeitshilfe für lokale Entscheidungsträger. Münster: Westfälische Wilhelms-Universität Münster.

Open Access Dieses Kapitel wird unter der Creative Commons Namensnennung 4.0 International Lizenz (http://creativecommons.org/licenses/by/4.0/deed.de) veröffentlicht, welche die Nutzung, Vervielfältigung, Bearbeitung, Verbreitung und Wiedergabe in jeglichem Medium und Format erlaubt, sofern Sie den/die ursprünglichen Autor(en) und die Quelle ordnungsgemäß nennen, einen Link zur Creative Commons Lizenz beifügen und angeben, ob Änderungen vorgenommen wurden.

Die in diesem Kapitel enthaltenen Bilder und sonstiges Drittmaterial unterliegen ebenfalls der genannten Creative Commons Lizenz, sofern sich aus der Abbildungslegende nichts anderes ergibt. Sofern das betreffende Material nicht unter der genannten Creative Commons Lizenz steht und die betreffende Handlung nicht nach gesetzlichen Vorschriften erlaubt ist, ist für die oben aufgeführten Weiterverwendungen des Materials die Einwilligung des jeweiligen Rechteinhabers einzuholen. 